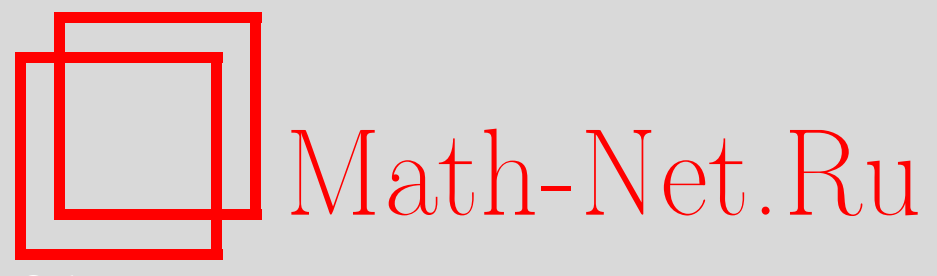

Б. А. Севастьянов, Асимптотика вероятности продолжения ограниченного снизу марковского критического ветвящегося процесса с непрерывным временем и бесконечной дисперсией, Дискрет. матем., 2006, том 18, выпуск $1,3-8$

DOI: https://doi.org/10.4213/dm28

Использование Общероссийского математического портала Math-Net.Ru подразумевает, что вы прочитали и согласны с пользовательским соглашением http://www . mathnet.ru/rus/agreement

Параметры загрузки:

IP : 54.174 .149 .18

26 апреля 2023 г., 14:10:48 


\title{
Асимптотика вероятности продолжения
}

ограниченного снизу марковского критического

ветвящегося процесса с непрерывным временем и бесконечной дисперсией

\author{
(ㄷ) 2006 г. $\quad$ Б. А. Севастьянов
}

Пусть $\mu(t)$ - число частиц в момент времени $t$ в критическом ветвящемся процессе с непрерывным временем. Известно, что при $t \rightarrow \infty$ вероятность продолжения процесса

$$
Q(t)=\mathbf{P}\{\mu(t)>0 \mid \mu(0)=1\} \rightarrow 0 .
$$

Отсюда следует, что

$$
Q_{m 0}=\mathbf{P}\{\mu(t)>0 \mid \mu(0)=m\} \sim m Q(t) \rightarrow 0
$$

при любом $m=2,3, \ldots$ При любых целых $m>r \geqslant 1$ введем обозначения

$$
Q_{m r}(t)=\mathbf{P}\left\{\inf _{0 \leqslant u \leqslant t} \mu(u)>r \mid \mu(0)=m\right\} .
$$

В статье доказывается, что при $t \rightarrow \infty$ имеет место асимптотика

$$
Q_{m r}(t) \sim(m-r) Q(t)
$$

для любого критического марковского ветвящегося процесса с непрерывным временем. Ранее этот результат был доказан для ветвящихся процессов с конечной дисперсией числа частиц.

Работа выполнена при поддержке Российского фонда фундаментальных исследований, проект 05.01.00035, и программы Президента Российской Федерации для поддержки ведущих научных школ, грант НШ-1758.2003.1.

Распределение числа частиц $\mu(t)$ в марковском ветвящемся процессе с непрерывным временем определяется производящей функцией

$$
f(s)=\sum_{n=0}^{\infty} p_{n} s^{n},
$$

где

$$
\begin{aligned}
\mathbf{P}\{\mu(t)=n \mid \mu(0)=1\} & =p_{n} t+o(t), \quad n \neq 1, \quad t \downarrow 0, \\
p_{1} & =-\sum_{n \neq 1} p_{n} .
\end{aligned}
$$


Производящая функщия

$$
F(t, s)=\sum_{k=0}^{\infty} \mathbf{P}\{\mu(t)=k \mid \mu(0)=1\} s^{k}
$$

является решением уравнения

$$
\frac{\partial F(t, s)}{\partial t}=f(F(t, s))
$$

при начальном условии

$$
F(0, s)=s
$$

(см. $[1,2])$.

Обозначим

$$
Q(t)=1-F(t, 0)=\mathbf{P}\{\mu(t)>0 \mid \mu(0)=1\}
$$

вероятность невырождения ветвящегося процесса $\mu(t)$ к времени $t$, если он начинается в момент $t=0$ с одной частицы, $\mu(0)=1$. Мы будем также называть $Q(t)$ вероятностью продолжения процесса в момент $t$.

Если

$$
f^{\prime}(1)=0, \quad f^{\prime \prime}(1) \neq 0,
$$

то процесс $\mu(t)$ называется критическим. Если

$$
f^{\prime \prime}(1)=b<\infty
$$

в критическом процессе $\mu(t)$, то дисперсия

$$
\mathbf{D} \mu(t)=b t
$$

конечна и при $t \rightarrow \infty$ вероятность

$$
Q(t) \sim \frac{2}{b t}
$$

(см. [2]).

Введем обозначение

$$
Q_{m r}(t)=\mathbf{P}\left\{\inf _{0 \leqslant u \leqslant t} \mu(u)>r \mid \mu(0)=m\right\} .
$$

Нетрудно видеть,что

$$
Q_{m 0}(t)=1-(1-Q(t))^{m} \sim m Q(t),
$$

когда $Q(t) \rightarrow 0$. Таким образом, в критическом процессе с конечной дисперсией

$$
Q_{m 0}(t) \sim \frac{2 m}{b t}, \quad t \rightarrow \infty .
$$


Вероятность (2) - это вероятность того, что ветвящийся процесс $\mu(t)$, начинающийся с $\mu(0)=m$, всегда имеет число частиц, большее $r$. Такой процесс мы будем называть ограниченным снизу.

Очевидно, что $Q_{m r}(t)$ убывает по $t$ и неравенство

$$
Q_{m r}(t)<Q_{m 0}(t)
$$

справедливо при любых $t>0$ и $m>r \geqslant 1$

В [3] показано, что при

$$
a=f^{\prime}(1)=0, \quad 0<b=f^{\prime \prime}(1)<\infty, \quad t \rightarrow \infty, \quad 1 \leqslant r<m
$$

имеет место асимптотика

$$
Q_{m r}(t) \sim \frac{2(m-r)}{b t} .
$$

В. М. Золотарев в [4] изучал асимптотическое поведение критического ветвящегося процесса $\mu(t)$ с бесконечной дисперсией $\mathrm{D} \mu(t)$. Он доказал, что при условии, что производящая функция $f(s)$ при $0<s \leqslant 1$ имеет вид

$$
f(1-s)=s^{1+\alpha} L(s),
$$

где $0<\alpha \leqslant 1, L(s)-$ медленно меняющаяся при $s \downarrow 0$ функция, вероятность $Q(t)$ продолжения процесса $\mu(t)$ при $t \rightarrow \infty$ имеет асимптотику

$$
Q(t) \sim \frac{L_{1}(t)}{t^{\beta}}
$$

где $\beta=1 / \alpha, L_{1}(t)$ - медленно меняющаяся при $t \rightarrow \infty$ функция, имеющая асимптотику

$$
L_{1}(t) \sim \frac{1}{\alpha^{\beta} L^{1 / \alpha}(Q(t))} .
$$

Цель этой статьи состоит в обобщении асимптотики (3) на случай бесконечной дисперсии $\mathbf{D} \mu(t)$.

Теорема 1. Пусть ветвящийся прочесс $\mu(t)$ определяется производящей функцией $f(s)$ вида (4), где $0<\alpha \leqslant 1, L(s)$ - медленно меняющаяся функчия при $s \downarrow 0$. Тогда вероятность $Q_{m r}(t), m>r \geqslant 1$, имеет при $t \rightarrow \infty$ асимптотику

$$
Q_{m r}(t) \sim(m-r) Q(t),
$$

где $Q(t) u L_{1}(t)$ определяются формулами (5) $u(6)$.

Для доказательства теоремы воспользуемся равенством

$$
\tau_{m 0}=\tau_{m r}+\tau_{r 0}
$$

где $\tau_{m 0}\left(\tau_{r 0}\right)$ - случайный момент вырождения процесса $\mu(t)$, начавшегося с $m(r)$ частиц, $\tau_{m r}-$ случайный момент первого достижения уровня $r$ для процесса, начавшегося с $m$ частиц, $\tau_{m r}$ и $\tau_{r 0}$ независимы. Обозначим $\varphi_{m r}(\lambda), \lambda \geqslant 0$, преобразование Лапласа $\tau_{m} r$. Так как

$$
Q_{m r}(t)=\mathbf{P}\left\{\tau_{m r}>t\right\}
$$


To

$$
\begin{aligned}
\varphi_{m r}(\lambda) & =-\int_{0}^{\infty} e^{-\lambda t} Q_{m r}(t) d t \\
& =1-\lambda \Phi_{m r}(\lambda)
\end{aligned}
$$

где

$$
\Phi_{m r}(\lambda)=\int_{0}^{\infty} e^{-\lambda t} Q_{m r}(t) d t
$$

Из (8) вытекает, что

$$
\varphi_{m r}(\lambda)=\frac{\varphi_{m 0}(\lambda)}{\varphi_{r 0}(\lambda)}
$$

откуда следует, что

$$
\Phi_{m r}(\lambda)=\frac{\Phi_{m 0}(\lambda)-\Phi_{r 0}(\lambda)}{1-\lambda \Phi_{r 0}(\lambda)}
$$

Далее нам понадобится тауберова теорема, которая является некоторым усилением теоремы 4 из книги Феллера (см. [5], гл. XIII, п. 5). Сформулируем ее в виде леммы.

Лемма 1. Пусть

$$
\begin{aligned}
\omega(\lambda) & =\int_{0}^{\infty} e^{-\lambda t} u(t) d t, \\
u(t) & =t^{l} v(t) \geqslant 0,
\end{aligned}
$$

где $v(t)$ - монотонная функция, $0<l<\infty, L(t)$ - медленно меняюшаяся функция при $t \rightarrow \infty$. В этом случае при $\lambda \downarrow 0$

$$
\omega(\lambda) \sim \frac{1}{\lambda^{l}} L\left(\frac{1}{\lambda}\right)
$$

тогда и только тогда, когда при $t \rightarrow \infty$

$$
u(t) \sim \frac{1}{\Gamma(l)} t^{l-1} L(t)
$$

Доказательство теоремы 1 . Обозначим $\rho$ и $\gamma$ целую и дробную части $\beta$. Из соотношения (4) в условии теоремы следует асимптотика (5) (см. [4]), которую можно записать в виде

$$
Q(t) \sim \frac{L_{1}(t)}{t^{\gamma}}
$$

Полагая в равенстве (9) $m=1, r=0$, получаем равенство

$$
\Phi(\lambda)=\int_{0}^{\infty} e^{-\lambda t} Q(t) d t
$$

где $\Phi(\lambda)=\Phi_{10}(\lambda), Q(t)=Q_{10}(t)$. Дифференцируя (12) $\rho$ раз по $\lambda$, получаем, что

$$
\Phi^{\rho}(\lambda)(-1)^{\rho}=\int_{0}^{\infty} e^{-\lambda t} t^{\rho} Q(t) d t .
$$


Используя в равенстве (13) асимптотику (11), по лемме получаем, что при $\lambda \downarrow 0$

$$
\Phi^{(\rho)}(\lambda)(-1)^{\rho} \sim \frac{\Gamma(1-\gamma)}{\lambda^{1-\gamma}} L_{1}\left(\frac{1}{\lambda}\right)
$$

Поскольку при $t \rightarrow \infty$

$$
\begin{aligned}
Q_{m 0}(t) & =1-(1-Q(t))^{m} \\
& \sim m Q(t) \sim \frac{m L_{1}(t)}{t^{\beta}},
\end{aligned}
$$

аналогично из

$$
\Phi_{m 0}(\lambda)=\int_{0}^{\infty} e^{-\lambda t} Q_{m 0}(t) d t
$$

при $\lambda \downarrow 0$ следует, что

$$
\Phi_{m 0}^{(\rho)}(\lambda)(-1)^{\rho} \sim \frac{m \Gamma(1-\gamma)}{\lambda^{1-\gamma}} L_{1}\left(\frac{1}{\lambda}\right)
$$

Аналогичным образом можно показать, что при любом целом $k, 0 \leqslant k<\rho$ и $\lambda \downarrow 0$

$$
\Phi_{m 0}^{(k)}(\lambda)(-1)^{k} \sim o\left(\frac{L_{1}\left(\lambda^{-1}\right)}{\lambda^{1-\gamma}}\right)
$$

Введем обозначения

$$
\begin{aligned}
& A(\lambda)=\Phi_{m 0}(\lambda)-\Phi_{r 0}(\lambda) \\
& B(\lambda)=\frac{1}{1-\lambda \Phi_{r 0}(\lambda)}
\end{aligned}
$$

Тогда

$$
\left.\Phi_{m r}^{(\rho)}(\lambda)(-1)^{\rho}=(-1)^{\rho}\left(A^{(\rho)}(\lambda) B(\lambda)+\sum_{k=0}^{\rho-1}\left(\begin{array}{l}
\rho \\
k
\end{array}\right) A^{(k)}(\lambda) B^{(\rho-k}\right)(\lambda)\right),
$$

где первое слагаемое при $\lambda \downarrow 0$

$$
(-1)^{\rho} A^{(\rho)}(\lambda) B(\lambda) \sim(m-r) \frac{\Gamma(1-\gamma)}{\lambda^{1-\gamma} L_{1}\left(\lambda^{-1}\right)},
$$

а остальные слагаемые имеют порядок $o\left(L_{1}\left(\lambda^{-1}\right) / \lambda^{1-\gamma}\right)$. Отсюда согласно лемме 1

$$
Q_{m r}(t) \sim(m-r) \frac{L_{1}(t)}{t^{\rho+\gamma}}
$$

что и доказывает теорему. 


\section{Список литературы}

1. Севастьянов Б. А., Теория ветвящихся случайных процессов. Успехи математических наук (1951) 6, №6, 47-99.

2. Севастьянов Б. А., Ветвящиеся прочессы. Наука, Москва, 1971.

3. Севастьянов Б. А., Ограниченные снизу ветвящиеся процессы. ДАН СССР (1978) 238, №4, $811-813$

4. Золотарев В. М., Уточнение ряда теорем теории ветвящихся случайных процессов. Теория вероятностей и ее применения (1957), 2, №2, 256-265.

5. Феллер В., Введение в теорию вероятностей и ее приложения, т. 2. Мир, Москва, 1967.

Статья поступила 10.11.2005. 